\title{
Erratum to: understanding how perceptions of tobacco constituents and the FDA relate to effective and credible tobacco risk messaging: a national phone survey of U.S. adults, 2014-2015
}

\author{
Marcella H. Boynton ${ }^{1,2}$, Robert P. Agans ${ }^{3,4}$, J. Michael Bowling ${ }^{1,2,3,4}$, Noel T. Brewer ${ }^{1,2}$, Erin L. Sutfin ${ }^{6}$, \\ Adam O. Goldstein ${ }^{2,5}$, Seth M. Noar ${ }^{2,7}$ and Kurt M. Ribis ${ }^{1,2^{*}}$
}

\section{Erratum}

As reported in the original paper [1], the Center for Regulatory Research on Tobacco Communication conducted a telephone survey in 2014-2015 with a national sample of adults ages 18 and older living in the United States $(N=5014)$. Poverty level was determined using the household size and income reported by the respondents and applying the federal poverty numbers available from the U.S. Department of Health and Human Services in 2014. A coding error was made during the data recoding process such that $2.7 \%$ of respondents $(n=129)$ were incorrectly classified as living above the poverty line. Below are updated Tables 1, 2 and 4 presenting both the original and corrected estimates. No substantive conclusions reported in the paper were affected by this correction.

\footnotetext{
Author details

'Department of Health Behavior, Gillings School of Global Public Health, University of North Carolina at Chapel Hill (UNC), CB \#7440, Chapel Hill, NC 27599-7440, USA. ${ }^{2}$ Lineberger Comprehensive Cancer Center, UNC, CB \#7295, Chapel Hill, NC 27599-7295, USA. ${ }^{3}$ Carolina Survey Research Laboratory, UNC, 730 Martin Luther King Jr Blvd, Chapel Hill, NC 27514, USA. ${ }^{4}$ Department of Biostatistics, Gillings School of Global Public Health, CB \#74203101 McGavran-Greenberg Hall, Chapel Hill, NC 27599-7420, USA. ${ }^{5}$ Department of Family Medicine, School of Medicine, UNC, CB \#7595Manning Drive, Chapel Hill, NC 27599-7595, USA. ${ }^{6}$ Department of Social Sciences and Health Policy, Wake Forest School of Medicine, Medical Center Boulevard, Winston-Salem, NC 27157, USA. ${ }^{7}$ School of Media and Journalism, UNC, CB \#3365, Chapel Hill, NC 27599-7440, USA.
}

Received: 11 January 2017 Accepted: 14 September 2017

Published online: 20 September 2017

\section{Reference}

1. Boynton M, Agans R, Bowling J, Brewer N, Sutfin E, Goldstein A, et al. Understanding how perceptions of tobacco constituents and the FDA relate to effective and credible tobacco risk messaging: A national phone survey of U.S. adults, 2014-2015. BMC Public Health. 2016;16(1). doi:10.1186/ s12889-016-3151-5.

2. US Census. American Community Survey United States Census Bureau. http://www.census.gov/acs. Accessed 21 Jan 2016

\footnotetext{
* Correspondence: kurt_ribisl@unc.edu

1 Department of Health Behavior, Gillings School of Global Public Health, University of North Carolina at Chapel Hill (UNC), CB \#7440, Chapel Hill, NC 27599-7440, USA

2Lineberger Comprehensive Cancer Center, UNC, CB \#7295, Chapel Hill, NC 27599-7295, USA
} 
Table 1 Demographic characteristics as compared to U.S. Census and other national surveys, CRRTC National Adult ( $\geq 18$ years) Phone Survey 2014-2015

\begin{tabular}{|c|c|c|c|c|}
\hline & \multirow{2}{*}{$\begin{array}{l}\text { Unweighted } \\
\%(n)\end{array}$} & \multicolumn{2}{|c|}{ Weighted } & \multirow{2}{*}{$\begin{array}{l}\text { National estimate } \\
\%\end{array}$} \\
\hline & & $\%$ & $95 \% \mathrm{Cl}$ & \\
\hline \multicolumn{5}{|l|}{ ORIGINALLY REPORTED ESTIMATES } \\
\hline \multicolumn{5}{|l|}{ Household Poverty } \\
\hline At or above federal poverty level & $84.0 \%(3901)$ & $85.7 \%$ & $(83.8-87.5)$ & $84.6 \%[2]$ \\
\hline Below federal poverty level & $16.0 \%(745)$ & $14.3 \%$ & $(12.5-16.2)$ & $15.4 \%[2]$ \\
\hline \multicolumn{5}{|l|}{ CORRECTED ESTIMATES } \\
\hline \multicolumn{5}{|l|}{ Household Poverty } \\
\hline At or above federal poverty level & $81.3 \%(3772)$ & $82.5 \%$ & $(80.4-84.7)$ & $84.6 \%[2]$ \\
\hline Below federal poverty level & $18.7 \%(868)$ & $17.5 \%$ & $(15.3-19.6)$ & $15.4 \%[2]$ \\
\hline
\end{tabular}

[2] US Census

Table 2 Percentage of smokers by selected demographic characteristics, CRRTC National Adult ( $\geq 18$ years) Phone Survey 2014-2015

\begin{tabular}{|c|c|c|c|c|}
\hline & \multicolumn{2}{|c|}{ Weighted } & \multicolumn{2}{|c|}{ National estimate } \\
\hline & $\%$ & $95 \% \mathrm{Cl}$ & $\%$ & $95 \% \mathrm{Cl}$ \\
\hline \multicolumn{5}{|l|}{ ORIGINALLY REPORTED ESTIMATES } \\
\hline \multicolumn{5}{|l|}{ Household Poverty } \\
\hline At or above federal poverty level & $15.4 \%$ & $(13.5-17.3)$ & $15.2 \%$ & $(14.6-15.9)$ \\
\hline Below federal poverty level & $29.3 \%$ & $(23.9-34.7)$ & $29.2 \%$ & $(27.5-31.0)$ \\
\hline \multicolumn{5}{|l|}{ CORRECTED ESTIMATES } \\
\hline \multicolumn{5}{|l|}{ Household Poverty } \\
\hline At or above federal poverty level & $15.1 \%$ & $(13.2-17.1)$ & $15.2 \%$ & $(14.6-15.9)$ \\
\hline Below federal poverty level & $27.9 \%$ & $(22.9-32.9)$ & $29.2 \%$ & $(27.5-31.0)$ \\
\hline
\end{tabular}


Table 4 Subset of Communication-related Variables - CRRTC National Adult Phone Survey 2014-2015

\begin{tabular}{|c|c|c|c|}
\hline & \multicolumn{3}{|c|}{ Weighted Proportion or M with 95\% Confidence Interval } \\
\hline & $\begin{array}{l}\text { ORIGINALLY REPORTED } \\
\text { ESTIMATES }\end{array}$ & $\begin{array}{l}\text { ORIGINALLY REPORTED } \\
\text { ESTIMATES }\end{array}$ & $\begin{array}{l}\text { CORRECTED } \\
\text { ESTIMATES }\end{array}$ \\
\hline & Total & Living in poverty & Living in poverty \\
\hline \multicolumn{4}{|l|}{ Information Seeking } \\
\hline \multicolumn{4}{|c|}{ Have you ever looked for information on chemicals in cigarettes and cigarette smoke? } \\
\hline Yes & $27.5 \%(25.4-29.7)$ & $25.7 \%(19.7-31.6)$ & $24.1 \%(18.8-29.4)$ \\
\hline No & $72.5 \%(70.3-74.6)$ & $74.3 \%(68.4-80.3)$ & $75.9 \%(70.6-81.2)$ \\
\hline \multicolumn{4}{|c|}{ In which 1 of these 3 places would you most like to see information on chemicals in cigarettes and cigarette smoke? } \\
\hline On cigarette packs & $54.8 \%(52.4-57.3)$ & $54.3 \%(47.4-61.2)$ & $54.6 \%(48.0-61.2)$ \\
\hline In stores & $15.0 \%(13.2-16.7)$ & $18.3 \%(13.2-23.5)$ & $18.3 \%(13.6-23.1)$ \\
\hline Online & $28.7 \%(26.5-30.9)$ & $25.5 \%(19.7-31.4)$ & $25.5 \%(20.2-30.9)$ \\
\hline Doesn't know, refused, or doesn't want information & $1.5 \%(0.9-2.08)$ & $1.8 \%(0.3-3.3)$ & $1.5 \%(0.2-2.7)$ \\
\hline \multicolumn{4}{|l|}{ Constituent Awareness } \\
\hline Aware of 0 of 4 constituents in cigarette smoke & $37.5 \%(35.0-40.1)$ & $43.1 \%(35.7-50.4)$ & $42.5 \%(35.5-49.5)$ \\
\hline Aware of 1 of 4 constituents in cigarette smoke & $35.8 \%(33.4-38.2)$ & $34.7 \%(28.2-41.2)$ & $33.6 \%(27.6-39.7)$ \\
\hline Aware of 2 of 4 constituents in cigarette smoke & $18.7 \%(16.7-20.7)$ & $13.6 \%(9.8-17.5)$ & $15.4 \%(9.3-21.6)$ \\
\hline Aware of 3 of 4 constituents in cigarette smoke & $5.6 \%(4.6-6.5)$ & $5.7 \%(3.0-8.4)$ & $4.9 \%(2.7-7.2)$ \\
\hline Aware of 4 of 4 constituents in cigarette smoke & $2.4 \%(1.7-3.1)$ & $2.9 \%(0.8-5.1)$ & $3.5 \%(1.4-5.7)$ \\
\hline \multicolumn{4}{|l|}{ Knowledge of and Trust for FDA and U.S. Federal Government } \\
\hline \multicolumn{4}{|l|}{ Have you ever heard of the FDA or Food and Drug Administration? } \\
\hline Yes & $94.6 \%(93.4-95.8)$ & $87.5 \%(83.0-92.0)$ & $87.1 \%(83.0-91.2)$ \\
\hline No & $5.4 \%(4.2-6.6)$ & $12.5 \%(8.0-17.0)$ & $12.9 \%(8.8-17.0)$ \\
\hline \multicolumn{4}{|l|}{ Can the FDA effectively regulate tobacco products? } \\
\hline Yes & $65.2 \%(62.6-67.8)$ & $67.8 \%(61.3-74.1)$ & $69.9 \%(63.9-75.9)$ \\
\hline No & $34.8 \%(32.2-37.4)$ & $32.2 \%(25.8-38.7)$ & $30.1 \%(24.1-36.1)$ \\
\hline $\begin{array}{l}\text { How much trust do you have in the federal government? M score, } \\
0=\text { none at all }-4=\text { a great deal }\end{array}$ & $2.0(1.9-2.0)$ & $2.2(2.0-2.4)$ & $3.1(2.9-3.2)$ \\
\hline
\end{tabular}

Note. Point estimates in bold text were found to be significantly different from their respective comparison group (e.g., smokers were compared to non-smokers, young adults compared to older adults, etc.) using either PROC SURVEYFREQ or PROC SURVEYREG to make the comparisons 